\title{
Robot-Assisted Endoscopic Resection: Current Status and Future Directions
}

\author{
Hung Leng Kaan ${ }^{1}$ and Khek Yu Ho \\ ${ }^{1}$ Department of General Surgery, National University Hospital, and ${ }^{2}$ Department of Medicine, Yong Loo Lin School of Medicine, National \\ University of Singapore, Singapore
}

Therapeutic endoscopic resection has gained favor for its ability to achieve high en bloc and histologically complete resection rates via a minimally invasive approach. The main technical difficulties faced by interventionists are first the lack of traction causing suboptimal visualization of the dissection field and second, the lack of triangulation using existing therapeutic apparatuses. These challenges can be overcome with the use of robots and the multiple degrees of freedom afforded by the robotic wrists. Nevertheless, complications such as bleeding and perforation can occur. It is hence beneficial for the robotic device to be equipped with additional abilities such as suturing. Once the robotic prototypes have been fully optimized and marketed, a structured program should be instituted to ensure proper and adequate training of the end-users. The future of robotics should then explore the possibility of developing a soft robot or a robot with more natural human-like movements. A robot with a force feedback mechanism would be superior and improve safety. Eventually, a supervised autonomous robot may perform interventions with greater precision and accuracy than an expert procedurist. This review describes the benefits of robot-assisted endoscopic resections, recent developments aimed at managing iatrogenic complications and future directions for robotic endoscopy. (Gut Liver 2020;14:150-152)

Key Words: Robotics; Endoscopy; Endoscopic submucosal dissection

\section{INTRODUCTION}

There are two main challenges faced by the endoscopist when performing endoscopic submucosal dissection (ESD). Firstly, using traditional resection instruments, there is no traction or counter-traction provided during ESD. Because of the lack of adequate traction, vision is compromised and this can lead to iatrogenic perforation and bleeding. ${ }^{1}$ Although skilled endoscopists might still be able to complete the endoscopic resection using superior dexterity gained from vast experience, unskilled endoscopists commonly find it technically demanding to complete the resection when they are unable to clearly visualize the dissection site.

Secondly, triangulation of the therapeutic equipment is not possible using the current endoscopic setup. Although therapeutics are gaining a more important role in the field of flexible endoscopy, therapeutic apparatuses for endoscopic intervention still need to be inserted through narrow-caliber channels which do not allow for triangulation. A white paper published by the joint committee of the American Society for Gastrointestinal Endoscopy and the Society of American Gastrointestinal and Endoscopic Surgeons emphasized the need to develop a novel multitasking triangulation platform which allows for complex endoscopic procedures to be performed. ${ }^{2}$

\section{HOW ROBOTIC ASSISTANCE CAN IMPROVE THERAPEU- TIC ENDOSCOPIC RESECTIONS}

The main benefits of robot-assisted ESD over conventional ESD is the improved maneuvering resulting from the increased degrees of freedom of the robotic wrists. Large external movements of the endoscopist can be scaled down and transformed to precise internal movements of the robotic wrists, hence improving ergonomics and allowing complex technical tasks to be completed. Robot-assisted procedures also allow for improved precision and accuracy because of superior three-dimensional dexterity. ${ }^{3}$

With these advantages in mind, the Master and Slave TransEndoluminal Robot (MASTER) (EndoMaster Pte Ltd., Singapore) was developed as a multitasking robotic endoscopic platform.

\footnotetext{
Correspondence to: Khek Yu Ho

Department of Medicine, Yong Loo Lin School of Medicine, National University of Singapore, NUHS Tower Block, 1E Kent Ridge Road, Level 11, Singapore 119228, Singapore

Tel: +65-6772-4362, Fax: +65-6779-4112, E-mail: mdchoky@nus.edu.sg

Received on February 7, 2019. Accepted on March 6, 2019. Published online June 4, 2019.

pISSN 1976-2283 eISSN 2005-1212 https://doi.org/10.5009/gnl19047

(a) This is an Open Access article distributed under the terms of the Creative Commons Attribution Non-Commercial License (http://creativecommons.org/licenses/by-nc/4.0) which permits unrestricted non-commercial use, distribution, and reproduction in any medium, provided the original work is properly cited.
} 
MASTER allows its operator to remotely control the endoscopic robot from a master console. ${ }^{4,5}$ Different end effectors (e.g., grasper, hook, injection needle, and electrocautery) can be inserted into the endoscopic channels. ${ }^{6}$ These instruments can then be used in triangulation and rotated like human wrists, because of the seven degrees of freedom afforded by the robotic wrists. This allows for optimal visualization and adequate traction of tissues during dissection. ${ }^{7-10}$ Additionally, the operation of the master console and robotic arms is intuitive and easy to use.

In a proof-of-concept study, MASTER was used to perform radical tonsillectomies on cadavers. ${ }^{11}$ The triangulation afforded by the robotic arms allowed the operator to perform bimanual tasks and the flexible endoscope allowed the camera to be manipulated into positions that a rigid camera system would not be able to reach.

Recently, a group in Korea developed a detachable robot, REXTER (revolute joint-based auxiliary transluminal endoscopic robot), which can be attached or detached from an existing general-use endoscope. ${ }^{12}$ They performed an in vitro test which demonstrated that safety could be greatly improved when unskilled operators used robotic assistance for therapeutic endoscopic resections.

\section{HOW WE CAN USE ROBOTICS TO MANAGE IATROGENIC COMPLICATIONS ARISING FROM ESD}

Because of the inherent complication of iatrogenic perforation in ESD, it is important that endoscopists are provided with reliable and easy-to-use endoscopic suturing devices. With this need in mind, we developed a novel suturing device using the MASTER system. The triangulation provided by the robot allows suturing and knot tying to be performed intuitively.

We conducted an animal study on an anaesthetized live pig using the novel suturing device. A double channel scope was inserted into the rectum. The computer controlled robotic arms were inserted into the working channels of the scope and controlled remotely via a master console. The right arm of the robot is a needle driver and the left arm of the robot is a tissue grasper. Both arms work in triangulation and they can be rotated $360^{\circ}$.

After the tissue incision was performed as part of ESD, the needle was loaded onto the needle driver and a figure of 8 suture was performed. Knots were then tied by passing the needle between suture loops formed by the robotic arms.

Through the pilot animal study, we demonstrated that our novel endoscopic robotic device can be used to suture perforations, and potentially suture bleeding sites and achieve hemostasis in ESD, without the need for additional surgical interventions. $^{13}$

\section{FUTURE DIRECTIONS FOR ROBOTIC ENDOSCOPY}

As the role of robot-assisted procedures continue to evolve and expand, it is paramount that training programs are instituted to "train-the-trainer." There should be a standardized curriculum and content validation prior to commencement of training for robot-assisted procedures. ${ }^{14}$ End-users must be adequately and properly trained prior to performing robot-assisted endoscopic resections in patients.

To improve ergonomics and reduce the learning curve associated with robot-assisted procedures, it would beneficial to improve robotic motions so that they become more natural and human-like. LaViers ${ }^{15}$ argued that no robot really moves like we do. The current robots outperform humans in force, speed and precision; but they are not able to move in myriad ways. The repertoire and diversity of robotic movements need to be expanded to allow for complex and variable motions, similar to what a human being is capable of. The latest generation of robots are soft robots ${ }^{16}$-they are constructed from elastic, flexible and compliant materials. This helps to increase the robot's range of motion. A soft robot can mimic an octopus and gently conform to the irregular surface of an object being held. Indeed, humanoid machines should move and gesture more like us.

Providing tactile force feedback information is also a leap forward in the right direction. The current limited or no force feedback system for robots can potentially lead to iatrogenic errors when performing therapeutic procedures. ${ }^{17,18}$ Force feedback during the manipulation of soft tissue is an important aspect of sensorimotor control of the human hand. A study by Wagner et al. ${ }^{19}$ showed that force feedback significantly reduced the magnitude of forces applied at the instrument tip during dissection. When force feedback was not available, subjects applied higher force levels over longer durations, and this translated to unnecessary trauma to the tissue being handled. These results held true across all levels of previous surgical training and experience.

Supervised autonomous robots performing with near humanlevel consciousness remains predominantly conceptual. ${ }^{20}$ Shademan et al. ${ }^{21}$ performed an in vivo supervised autonomous soft tissue surgery in an open surgical setting using a computer program preloaded with best human surgical practices. The program generated a plan to complete complex surgical tasks on soft tissue, such as suturing and anastomosis. The results showed that supervised autonomous procedures performed by the robot were superior to even surgeries performed by expert surgeons, in terms of consistency of suturing, average suture space, number of mistakes made, completion time and pressure at which anastomosis leaked. This study demonstrated the potential for supervised autonomous robots in improving the efficacy and outcome measures of interventional procedures. 


\section{CONCLUSION}

The field of robot-assisted endoscopic resection is exciting and developing at a fast pace. The main benefits of using a robot to assist endoscopic resection are to provide triangulation of instruments and optimal visualization of the dissection field from adequate traction of resected tissues. Nevertheless, with the introduction of robotics in endoscopy, it is imperative that additional measures are put it place to allow the robot to manage complications such as iatrogenic perforation and bleeding. We believe that we have developed a novel robotic endoscopic device that can suture perforations resulting from ESD without the need for additional surgical intervention. Once these robotic devices have been marketed, proper programs must be instituted to ensure that end-users are adequately trained. In the future, the areas of interest for further innovation in the field of robotic endoscopy include providing force feedback, developing a soft robot that moves more naturally, and eventually, designing a supervised autonomous robot.

\section{CONFLICTS OF INTEREST}

K.Y.H. is the co-founder of EndoMaster Pte Ltd., Singapore. No other potential conflict of interest relevant to this article was reported.

\section{ORCID}

Khek Yu Ho

https://orcid.org/0000-0002-2932-1962

\section{REFERENCES}

1. Saito Y, Sumiyama K, Chiu PW. Robot assisted tumor resection devices. Expert Rev Med Devices 2017;14:657-662.

2. ASGE; SAGES. ASGE/SAGES Working Group on Natural Orifice Translumenal Endoscopic Surgery White Paper October 2005. Gastrointest Endosc 2006;63:199-203.

3. Ashrafian H, Clancy 0, Grover V, Darzi A. The evolution of robotic surgery: surgical and anaesthetic aspects. Br J Anaesth 2017;119:i72-i84.

4. Phee SJ, Low SC, Huynh VA, Kencana AP, Sun ZL, Yang K. Master and slave transluminal endoscopic robot (MASTER) for natural orifice transluminal endoscopic surgery (NOTES). Conf Proc IEEE Eng Med Biol Soc 2009;2009:1192-1195.

5. Wong JY, Ho KY. Robotics for advanced therapeutic colonoscopy. Clin Endosc 2018;51:552-557.

6. Phee SJ, Ho KY, Lomanto D, et al. Natural orifice transgastric endoscopic wedge hepatic resection in an experimental model using an intuitively controlled master and slave transluminal endoscopic robot (MASTER). Surg Endosc 2010;24:2293-2298.

7. Wang Z, Phee SJ, Lomanto D, et al. Endoscopic submucosal dissection of gastric lesions by using a master and slave transluminal endoscopic robot: an animal survival study. Endoscopy 2012;44:690-694.

8. Ho KY, Phee SJ, Shabbir A, et al. Endoscopic submucosal dissection of gastric lesions by using a master and slave transluminal endoscopic robot (MASTER). Gastrointest Endosc 2010;72:593599.

9. Phee SJ, Reddy N, Chiu PW, et al. Robot-assisted endoscopic submucosal dissection is effective in treating patients with early-stage gastric neoplasia. Clin Gastroenterol Hepatol 2012;10:1117-1121.

10. Chiu PW, Phee SJ, Wang Z, et al. Feasibility of full-thickness gastric resection using master and slave transluminal endoscopic robot and closure by Overstitch: a preclinical study. Surg Endosc 2014;28:319-324.

11. Tay G, Tan HK, Nguyen TK, Phee SJ, Iyer NG. Use of the EndoMaster robot-assisted surgical system in transoral robotic surgery: a cadaveric study. Int J Med Robot 2018;14:e1930.

12. Kim BG, Choi HS, Park SH, et al. A pilot study of endoscopic submucosal dissection using an endoscopic assistive robot in a porcine stomach model. Gut Liver 2019;13:402-408.

13. Cao L, Li XG, Phan PT, et al., "Sewing up the wound: a robotic suturing system for flexible endoscopy.” IEEE Robot Autom Mag. Forthcoming 2020.

14. Collins JW, Levy J, Stefanidis D, et al. Utilising the Delphi process to develop a proficiency-based progression train-the-trainer course for robotic surgery training. Eur Urol 2019;75:775-785.

15. LaViers A. Make robot motions natural. Nature 2019;565:422-424.

16. Calisti M, Picardi G, Laschi C. Fundamentals of soft robot locomotion. J R Soc Interface 2017;14:20170101.

17. Shi Y, Zhou C, Xie L, et al. Research of the master-slave robot surgical system with the function of force feedback. Int J Med Robot 2017;13:e1826.

18. Zhao B, Nelson CA. A sensorless force-feedback system for robotassisted laparoscopic surgery. Comput Assist Surg (Abingdon) 2019;24(Suppl 1):36-43.

19. Wagner CR, Stylopoulos N, Jackson PG, Howe RD. The benefit of force feedback in surgery: examination of blunt dissection. Presence (Camb) 2007;16:252-262.

20. Ashrafian H, Darzi A, Athanasiou T. A novel modification of the Turing test for artificial intelligence and robotics in healthcare. Int J Med Robot 2015;11:38-43.

21. Shademan A, Decker RS, Opfermann JD, Leonard S, Krieger A, Kim PC. Supervised autonomous robotic soft tissue surgery. Sci Transl Med 2016;8:337ra64. 